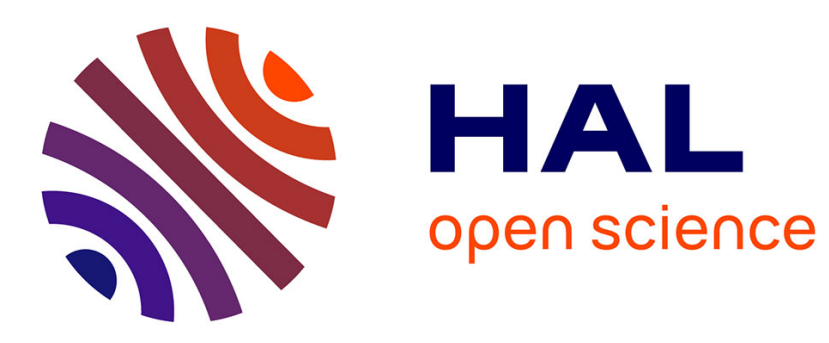

\title{
Asteroid close encounters characterization using differential algebra: the case of Apophis
}

R. Armellin, P. Di Lizia, F. Bernelli-Zazzera, M. Berz

\section{To cite this version:}

R. Armellin, P. Di Lizia, F. Bernelli-Zazzera, M. Berz. Asteroid close encounters characterization using differential algebra: the case of Apophis. Celestial Mechanics and Dynamical Astronomy, 2010, 107 (4), pp.451-470. 10.1007/s10569-010-9283-5 . hal-00552520

\section{HAL Id: hal-00552520 \\ https://hal.science/hal-00552520}

Submitted on 6 Jan 2011

HAL is a multi-disciplinary open access archive for the deposit and dissemination of scientific research documents, whether they are published or not. The documents may come from teaching and research institutions in France or abroad, or from public or private research centers.
L'archive ouverte pluridisciplinaire HAL, est destinée au dépôt et à la diffusion de documents scientifiques de niveau recherche, publiés ou non, émanant des établissements d'enseignement et de recherche français ou étrangers, des laboratoires publics ou privés. 


\title{
Asteroid close encounters characterization using differential algebra: the case of Apophis
}

\author{
R. Armellin • P. Di Lizia · F. Bernelli-Zazzera • \\ M. Berz
}

Received: 26 June 2009 / Revised: 22 April 2010 / Accepted: 23 April 2010 /

Published online: 13 June 2010

(C) Springer Science+Business Media B.V. 2010

\begin{abstract}
A method for the nonlinear propagation of uncertainties in Celestial Mechanics based on differential algebra is presented. The arbitrary order Taylor expansion of the flow of ordinary differential equations with respect to the initial condition delivered by differential algebra is exploited to implement an accurate and computationally efficient Monte Carlo algorithm, in which thousands of pointwise integrations are substituted by polynomial evaluations. The algorithm is applied to study the close encounter of asteroid Apophis with our planet in 2029. To this aim, we first compute the high order Taylor expansion of Apophis' close encounter distance from the Earth by means of map inversion and composition; then we run the proposed Monte Carlo algorithm to perform the statistical analysis.
\end{abstract}

Keywords Uncertainties propagation · Monte Carlo simulation ·

Apophis close encounter - Differential algebra

\section{Introduction}

The propagation of uncertainties in orbital mechanics is usually addressed by linear propagation models (Battin 1968; Crassidis and Junkins 2004; Montenbruck and Gill 2001) or full nonlinear Monte Carlo simulations (Maybeck 1982). The main advantage of the linear methods is the simplification of the problem, but their accuracy drops off for highly nonlinear

R. Armellin · P. Di Lizia $(\varangle) \cdot$ F. Bernelli-Zazzera

Dipartimento di Ingegneria Aerospaziale, Politecnico di Milano, Via La Masa, 34, 20156 Milano, Italy

e-mail: dilizia@aero.polimi.it

R. Armellin

e-mail: armellin@aero.polimi.it

F. Bernelli-Zazzera

e-mail: bernelli@aero.polimi.it

M. Berz

Department of Physics and Astronomy, Michigan State University, East Lansing, MI 48824, USA

e-mail: berz@msu.edu 
systems and/or long time propagations. On the other hand, Monte Carlo simulations provide true trajectory statistics, but are computationally intensive. The tools currently used for the robust detection and prediction of planetary encounters and potential impacts of Near Earth Objects (NEO) are based on these techinques (Chesley and Milani 2000; Chodas and Yeomans 1999; Milani et al. 2000), and thus suffer the same limitations. The effect of the coordinate system on the propagated statistics is analyzed by Junkins et al. (1996) and Junkins and Singla (2004) and used to develop an alternative approach to orbit uncertainty propagation. However, this method is based on a linear assumption and thus cannot map nonlinearities. An alternate way to analyze trajectory statistics by incorporating higher-order Taylor series terms that describe localized nonlinear motion is proposed by Park and Scheeres (2006). Their approach is based on proving the integral invariance of the probability density function via solutions of the Fokker-Planck equations for diffusionless systems, and by combining this result with the nonlinear state propagation to derive an analytic representation of the nonlinear uncertainty propagation. As a result, the method enables the nonlinear mapping of Gaussian statistics, bypassing the drawbacks of Monte Carlo simulations. However, it is limited to systems derived from a single potential.

Differential algebraic (DA) techniques are proposed as a valuable tool to develop an alternative approach to tackle the previous tasks. Differential algebra supplies the tools to compute the derivatives of functions within a computer environment (Berz 1999a,b; Berz and Makino 2006). More specifically, by substituting the classical implementation of real algebra with the implementation of a new algebra of Taylor polynomials, any function $f$ of $n$ variables is expanded into its Taylor polynomial up to an arbitrary order $k$. This has an important consequence when the numerical integration of an ordinary differential equation (ODE) is performed by means of an arbitrary integration scheme. Any explicit integration scheme is based on algebraic operations, involving the evaluation of the ODE right hand side at several integration points. Therefore, starting from the DA representation of the initial condition and carrying out all the evaluations in the DA framework, the flow of an ODE is obtained at each step as its Taylor expansion in the initial condition (Di Lizia et al. 2008). The availability of such high order expansions is exploited when problems with uncertain initial conditions have to be analyzed. As the accuracy of the Taylor expansion can be kept arbitrarily high by adjusting the expansion order, the approach of classical Monte Carlo simulations can be enhanced by replacing thousands of integrations with evaluations of the Taylor expansion of the flow. As a result, the computational time reduces considerably without any significant loss in accuracy.

The algorithm is applied to the prediction of Apophis planetary encounter and potential impact, taking into account its measurement uncertainties. The availability of high order maps in space and time, and intrinsic tools for their inversion, are exploited to reduce the computation of the close encounter distance (CED) from the Earth of all the asteroids belonging to the initial uncertainty cloud (commonly referred to as virtual asteroids; Milani et al. 2002) to the simple evaluation of polynomials. Similar techniques exploiting high order Taylor expansions of the flow of ODE and their inverses obtained with DA techniques have already been efficiently utilized in beam physics. Two noticeable applications are the reconstruction of trajectories in particle spectrographs together with the reconstructive correction of residual aberrations (Berz et al. 1993), and the end-to-end simulations of fragment separators (Erdelyi et al. 2007). This paper presents an application to Celestial Mechanics.

The paper is organized as follows. Sections 2 and 3 contain a brief introduction to differential algebra and some hints on how high order expansion of the flow can be obtained. These techniques are then applied to obtain the flow expansion of Apophis' dynamics. The 
DA-based Monte Carlo algorithm is then introduced and utilized to perform the statistical analysis of Apophis CED in 2029. Some final remarks conclude the paper.

\section{Notes on differential algebra}

DA techniques, exploited here to obtain $k$-th order Taylor expansions of the flow of a set of ODE with respect to the initial condition, find their origin in the attempt to solve analytical problems by an algebraic approach (Berz 1999b). Historically, the treatment of functions in numerics has been based on the treatment of numbers, and the classical numerical algorithms are based on the mere evaluation of functions at specific points. DA techniques rely on the observation that it is possible to extract more information on a function rather than its mere values. The basic idea is to bring the treatment of functions and the operations on them to the computer environment in a similar way as the treatment of real numbers. Referring to Fig. 1, consider two real numbers $a$ and $b$. Their transformation into the floating point representation, $\bar{a}$ and $\bar{b}$, respectively, is performed to operate on them in a computer environment. Then, given any operation $*$ in the set of real numbers, an adjoint operation $\circledast$ is defined in the set of floating point (FP) numbers so that the diagram in Fig. 1 commutes. (The diagram commutes approximately in practice due to truncation errors.) Consequently, transforming the real numbers $a$ and $b$ into their FP representation and operating on them in the set of FP numbers returns the same result as carrying out the operation in the set of real numbers and then transforming the achieved result in its FP representation. In a similar way, let us suppose two $k$ differentiable functions $f$ and $g$ in $n$ variables are given. In the framework of differential algebra, the computer operates on them using their $k$-th order Taylor expansions, $F$ and $G$, respectively. Therefore, the transformation of real numbers in their FP representation is now substituted by the extraction of the $k$-th order Taylor expansions of $f$ and $g$. For each operation in the space of $k$ differentiable functions, an adjoint operation in the space of Taylor polynomials is defined so that the corresponding diagram commutes; i.e., extracting the Taylor expansions of $f$ and $g$ and operating on them in the space of Taylor polynomials (labeled as ${ }_{k} D_{n}$ ) returns the same result as operating on $f$ and $g$ in the original space and then extracting the Taylor expansion of the resulting function.

The straightforward implementation of differential algebra in a computer allows to compute the Taylor coefficients of a function up to a specified order $k$, along with the function evaluation, with a fixed amount of effort. The Taylor coefficients of order $k$ for sums and product of functions, as well as scalar products with reals, can be computed from those of summands and factors; therefore, the set of equivalence classes of functions can be endowed with well-defined operations, leading to the so-called truncated power series algebra (Berz 1986, 1987). Similarly to the algorithms for floating point arithmetic, the algorithms for functions followed, including methods to perform composition of functions, to invert them, to
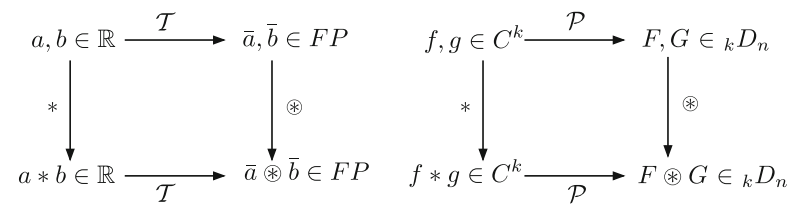

Fig. 1 Analogy between the floating point representation of real numbers in a computer environment (left figure) and the introduction of the algebra of Taylor polynomials in the differential algebraic framework (right figure) 
solve nonlinear systems explicitly, and to treat common elementary functions (Berz 1999a,b). In addition to these algebraic operations, the DA framework is endowed with differentiation and integration operators, therefore finalizing the definition of the DA structure. The differential algebra sketched in this section was implemented in the software COSY-Infinity (Berz and Makino 2006).

\subsection{The minimal differential algebra}

The key feature of differential algebra is that it enables the automatic computation of derivatives in a computer environment. In this section the simplest nontrivial differential algebra is introduced to present an outline on the basic concepts that its implementation relies on. For a detailed description refer to Berz (1999b), where the extension to arbitrary order and multivariate functions is discussed.

Consider two ordered pairs $\left(q_{0}, q_{1}\right),\left(r_{0}, r_{1}\right)$, and a scalar $t$, with $q_{0}, q_{1}, r_{0}, r_{1}$, and $t$ real numbers. Define the addition "+" and the multiplication "." by a scalar and between two pairs as:

$$
\begin{aligned}
\left(q_{0}, q_{1}\right)+\left(r_{0}, r_{1}\right) & =\left(q_{0}+r_{0}, q_{1}+r_{1}\right) \\
t \cdot\left(q_{0}, q_{1}\right) & =\left(t q_{0}, t q_{1}\right) \\
\left(q_{0}, q_{1}\right) \cdot\left(r_{0}, r_{1}\right) & =\left(q_{0} r_{0}, q_{0} r_{1}+q_{1} r_{0}\right) .
\end{aligned}
$$

The ordered pairs with the introduced arithmetic are referred to as ${ }_{1} D_{1}$. The multiplication of vectors is seen to have $(1,0)$ as the unity element. The multiplication is commutative, associative, and distributive with respect to addition. Together, the three operations defined in (1) form an algebra. Furthermore, they do form an extension of real numbers, as $(r, 0)+(s, 0)=(r+s, 0)$ and $(r, 0) \cdot(s, 0)=(r s, 0)$, so that the reals can be included. However ${ }_{1} D_{1}$ is not a field, as $\left(q_{0}, q_{1}\right)$ has a multiplicative inverse in ${ }_{1} D_{1}$ if and only if $q_{0} \neq 0$. If $q_{0} \neq 0$ then

$$
\left(q_{0}, q_{1}\right)^{-1}=\left(\frac{1}{q_{0}},-\frac{q_{1}}{q_{0}^{2}}\right) .
$$

The algebra in ${ }_{1} D_{1}$ becomes a differential algebra by introducing a map $\partial$ from ${ }_{1} D_{1}$ to itself, and proving that the map is a derivation. Define $\partial:{ }_{1} D_{1} \rightarrow{ }_{1} D_{1}$ by

$$
\partial\left(q_{0}, q_{1}\right)=\left(0, q_{1}\right) .
$$

Note that

$$
\begin{aligned}
\partial\left\{\left(q_{0}, q_{1}\right)+\left(r_{0}, r_{1}\right)\right\} & =\partial\left(q_{0}+r_{0}, q_{1}+r_{1}\right)=\left(0, q_{1}+r_{1}\right) \\
& =\left(0, q_{1}\right)+\left(0, r_{1}\right)=\partial\left(q_{0}, q_{1}\right)+\partial\left(r_{0}, r_{1}\right)
\end{aligned}
$$

and

$$
\begin{aligned}
\partial\left\{\left(q_{0}, q_{1}\right) \cdot\left(r_{0}, r_{1}\right)\right\} & =\partial\left(q_{0} r_{0}, q_{0} r_{1}+q_{1} r_{0}\right)=\left(0, q_{0} r_{1}+q_{1} r_{0}\right) \\
& =\left(0, q_{1}\right) \cdot\left(r_{0}, r_{1}\right)+\left(0, r_{1}\right) \cdot\left(q_{0}, q_{1}\right) \\
& =\partial\left\{\left(q_{0}, q_{1}\right)\right\} \cdot\left(r_{0}, r_{1}\right)+\left(q_{0}, q_{1}\right) \cdot \partial\left\{\left(r_{0}, r_{1}\right)\right\}
\end{aligned}
$$

This holds for all $\left(q_{0}, q_{1}\right),\left(r_{0}, r_{1}\right) \in{ }_{1} D_{1}$. The $\partial$ operator is linear over addition and obeys the Leibniz rule over the algebra multiplication, thus it is a derivation and $\left({ }_{1} D_{1}, \partial\right)$ is a differential algebra. 
The most important aspect of ${ }_{1} D_{1}$ is that it allows the automatic computation of derivatives. Let us assume to have two functions $f$ and $g$ and to put their values and their derivatives at the origin in the form $\left(f(0), f^{\prime}(0)\right)$ and $\left(g(0), g^{\prime}(0)\right)$; i.e., as two vectors in ${ }_{1} D_{1}$. If the derivative of the product $f g$ is of interest, it has just to be looked at the second component of the product $\left(f(0), f^{\prime}(0)\right) \cdot\left(g(0), g^{\prime}(0)\right)$; whereas the first component gives the value of the product of the functions. Therefore, if two vectors contain the values and the derivatives of two functions, their product contains the values and the derivatives of the product function.

Defining the operation [ ] from the space of differential functions to ${ }_{1} D_{1}$ via

$$
[f]=\left(f(0), f^{\prime}(0)\right),
$$

it holds

$$
\begin{aligned}
{[f+g] } & =[f]+[g] \\
{[f g] } & =[f] \cdot[g]
\end{aligned}
$$

and

$$
[1 / g]=[1] /[g]=1 /[g]
$$

by using (2). This observation can be used to compute derivatives of many kinds of functions algebraically by means of the arithmetic rules on ${ }_{1} D_{1}$, starting from applying the operator [ ] to the identity function

$$
[x]=(x, 1) .
$$

Note that this is equivalent to extract the coefficients of the first order Taylor expansion of the identity function; i.e., $[x]=(x, 1)=x+\delta x$.

Consider the example

$$
f(x)=\frac{1}{x+\frac{1}{x}}
$$

and its derivative

$$
f^{\prime}(x)=\frac{\left(1 / x^{2}\right)-1}{(x+(1 / x))^{2}} .
$$

The function value and its derivative at the point $x=3$ are

$$
f(3)=\frac{3}{10}, \quad f^{\prime}(3)=-\frac{2}{25} .
$$

If the function (10) is evaluated in ${ }_{1} D_{1}$ by substituting $x$ with its DA at 3 ; i.e., $(3,1)=3+\delta x$, it results

$$
\begin{aligned}
f((3,1)) & =\frac{1}{(3,1)+1 /(3,1)}=\frac{1}{(3,1)+(1 / 3,-1 / 9)} \\
& =\frac{1}{(10 / 3,8 / 9)}=\left(\frac{3}{10},-\frac{8}{9} / \frac{100}{9}\right)=\left(\frac{3}{10},-\frac{2}{25}\right) .
\end{aligned}
$$


As it can be seen after the evaluation of the function, the first element of the result is the value of the function at $x=3$, whereas the second is the value of the derivative of the function at $x=3$. This result is simply justified by applying the relations (7) and (8)

$$
\begin{aligned}
{[f(x)] } & =\left[\frac{1}{x+1 / x}\right]=\frac{1}{[x+1 / x]} \\
& =\frac{1}{[x]+[1 / x]}=\frac{1}{[x]+1 /[x]} \\
& =f([x]) .
\end{aligned}
$$

The method can be generalized to allow the treatment of common intrinsic functions like sin and exp. This differential algebra can be straightforwardly implemented on the computer by exploiting operation overloading.

\section{High order expansion of the flow}

The extension of the differential algebra introduced in Sect. 2.1 to ${ }_{k} D_{n}$ allows the derivatives of any function $f$ of $n$ variables to be computed up to an arbitrary order $k$, along with the function evaluation. This has an important consequence when the numerical integration of an ODE is performed by means of an arbitrary integration scheme. Any explicit integration scheme is based on algebraic operations, involving the evaluation of the ODE right hand side at several integration points. Therefore, carrying out all the evaluations in the DA framework allows differential algebra to compute the arbitrary order expansion of the flow of a general ODE with respect to the initial condition.

Without loss of generality, consider the scalar initial value problem

$$
\left\{\begin{array}{l}
\dot{x}=f(x, t) \\
x\left(t_{0}\right)=x_{0}
\end{array}\right.
$$

and the associated phase flow $\varphi\left(t ; x_{0}\right)$. We now want to show that, starting from the DA representation of the initial condition $x_{0}$, differential algebra allows us to propagate the Taylor expansion of the flow in $x_{0}$ forward in time, up to the final time $t_{f}$.

Replace the point initial condition $x_{0}$ with the DA representative of its identity function up to order $k$, which is a $(k+1)$-tuple of Taylor coefficients. (Note that $x_{0}$ is the flow evaluated at the initial time; i.e, $x_{0}=\varphi\left(t_{0} ; x_{0}\right)$.) As for the identity function only the first two coefficients, corresponding to the constant part and the first derivative respectively, are non zeros, we can write $\left[x_{0}\right]$ as $x_{0}+\delta x_{0}$, in which $x_{0}$ is the reference point for the expansion. If all the operations of the numerical integration scheme are carried out in the framework of differential algebra, the phase flow $\varphi\left(t ; x_{0}\right)$ is approximated, at each fixed time step $t_{i}$, as a Taylor expansion in $x_{0}$.

As an example, consider the forward Euler's scheme

$$
x_{i}=x_{i-1}+f\left(x_{i-1}\right) \Delta t
$$

and substitute the initial value with the DA identity $\left[x_{0}\right]=x_{0}+\delta x_{0}$. At the first time step we have

$$
\left[x_{1}\right]=\left[x_{0}\right]+f\left(\left[x_{0}\right]\right) \cdot \Delta t .
$$


If the function $f$ is evaluated in the DA framework, the output of the first step, $\left[x_{1}\right]$, is the $k$-th order Taylor expansions of the flow $\varphi\left(t ; x_{0}\right)$ in $x_{0}$ for $t=t_{1}$. Note that, as a result of the DA evaluation of $f\left(\left[x_{0}\right]\right)$, the $(k+1)$-tuple $\left[x_{1}\right]$ may include several non zeros coefficients corresponding to high order terms in $\delta x_{0}$. The previous procedure can be inferred through the subsequent steps. The result of the final step is the $k$-th order Taylor expansion of $\varphi\left(t ; x_{0}\right)$ in $x_{0}$ at the final time $t_{f}$. Thus, the flow of a dynamical system can be approximated, at each time step $t_{i}$, as a $k$-th order Taylor expansion in $x_{0}$ in a fixed amount of effort.

Integration schemes based on DA pave the way to the nonlinear mapping of uncertainties investigated in this paper. A first example is presented hereafter about the propagation of errors on initial conditions. The Taylor polynomials resulting from the use of DA-based numerical integrators expand the solution of the initial value problem (15) with respect to the initial condition. Thus, the dependence of the solution $x(t)$ with respect to the initial condition is available, at a time $t_{i}$, in terms of a $k$-th order polynomial map $\mathcal{M}_{x_{0}}\left(\delta x_{0}\right)$, where $\delta x_{0}$ represents the displacement from the reference initial condition. The evaluation of the map $\mathcal{M}_{x_{0}}\left(\delta x_{0}\right)$ for a selected value of $\delta x_{0}$ supplies the $k$-th order Taylor approximation of the solution $x(t)$ at $t_{i}$ corresponding to the displaced initial condition. The accuracy of the result depends on the expansion order $k$ and the value of the displacement $\delta x_{0}$. The main advantage of the DA-based approach is that the new solution is obtained by evaluating a polynomial map, thus avoiding any additional numerical integration. Consequently, if many values of $\delta x_{0}$ are to be processed, multiple polynomial evaluations can be efficiently performed in place of multiple intensive numerical integrations. Based on this observation, we introduce a DA-based Monte Carlo algorithm, whose performances are assessed using Apophis close encounter with the Earth in 2029 as test case.

As a final remark, it is worth noting that methods to obtain high order expansions of the flow of ODE have been already explored in detail by Griffith et al. (2004) and Park and Scheeres $(2006,2007)$. These authors have shown the potentials of these techniques by applying them to the development of high order methods for the solution of relevant spacerelated problems such as low-thrust Earth-Mars transfers, spacecraft targeting in two-body and Hill three-body dynamics, and trajectory estimation in the circular restricted three-body problem. It has to be stressed that their approach firstly requires to derive the ODE for the so-called state transition tensors and secondly to integrate them along with the reference solution, a technique more commonly known as solving the variational equations. On the other hand, it is not required to write any additional set of ODE within the differential algebraic approach, being the arbitrary high order expansion of the flow a straightforward result of the implemented algebra.

\section{DA integration of Apophis dynamics}

\subsection{Dynamical models}

The study of the motion of a NEO in the Solar System with an accuracy sufficient to predict collisions requires the inclusion of various relativistic corrections to the well-known Newtonian forces based on the Kepler's force law. Specifically, the full equation of motion in the Solar System including the relevant relativistic effects is given by 


$$
\begin{aligned}
\ddot{\boldsymbol{r}}= & G \sum_{i} \frac{m_{i}\left(\boldsymbol{r}_{i}-\boldsymbol{r}\right)}{r_{i}^{3}}\left\{1-\frac{2(\beta+\gamma)}{c^{2}} G \sum_{j} \frac{m_{j}}{r_{j}}-\frac{2 \beta-1}{c^{2}} G \sum_{j \neq i} \frac{m_{j}}{r_{i j}}+\frac{\gamma|\dot{\boldsymbol{r}}|^{2}}{c^{2}}\right. \\
& \left.+\frac{(1+\gamma)\left|\dot{\boldsymbol{r}}_{i}\right|^{2}}{c^{2}}-\frac{2(1+\gamma)}{c^{2}} \dot{\boldsymbol{r}} \cdot \dot{\boldsymbol{r}}_{i}-\frac{3}{2 c^{2}}\left[\frac{\left(\boldsymbol{r}-\boldsymbol{r}_{i}\right) \cdot \dot{\boldsymbol{r}}_{i}}{r_{i}}\right]^{2}+\frac{1}{2 c^{2}}\left(\boldsymbol{r}_{i}-\boldsymbol{r}\right) \cdot \ddot{\boldsymbol{r}}_{i}\right\} \\
& +G \sum_{i} \frac{m_{i}}{c^{2} r_{i}}\left\{\frac{3+4 \gamma}{2} \ddot{\boldsymbol{r}}_{i}+\frac{\left\{\left[\boldsymbol{r}-\boldsymbol{r}_{i}\right] \cdot\left[(2+2 \gamma) \dot{\boldsymbol{r}}-(1+2 \gamma) \dot{\boldsymbol{r}}_{i}\right]\right\}\left(\dot{\boldsymbol{r}}-\dot{\boldsymbol{r}}_{i}\right)}{r_{i}^{2}}\right\},
\end{aligned}
$$

where $\boldsymbol{r}$ is the point of interest, $G$ is the gravitational constant; $m_{i}$ and $\boldsymbol{r}_{i}$ are the mass and the Solar System barycentric position of body or planetary system $i ; r_{i}=\left|\boldsymbol{r}_{i}-\boldsymbol{r}\right| ; c$ is the speed of light in vacuum; and $\beta$ and $\gamma$ are the parametrized post-Newtonian parameters measuring the nonlinearity in superposition of gravity and space curvature produced by unit rest mass (Seidelmann 1992).

In Eq. 18 it is assumed that the object we are integrating is affected by the gravitational attraction of $n$ bodies, but has no gravitational effect on them; i.e., we are adopting the restricted $(n+1)$-body problem approximation. The positions, velocities, and accelerations of the $n$ bodies are considered as given values, computed by cubic spline interpolations of data retrieved from HORIZONS Web-Interface (http://ssd.jpl.nasa.gov/horizons.cgi). These interpolations are necessary as in the DA framework all the computations must be performed within COSY-Infinity and the use of external code is not permitted. The cubic splines are built so as to keep the maximum error with respect to HORIZONS' ephemerides of the order of $10^{-9} \mathrm{AU}$ and $10^{-10} \mathrm{AU} /$ day for bodies' position and velocity, respectively (see BernelliZazzera et al. (2009) for details). In our integrations $n$ includes the Sun, planets, the Moon, Ceres, Pallas, and Vesta. For planets with moons, with the exception of the Earth, the center of mass of the system is considered. The dynamical model is written in the J2000.0 Ecliptic reference frame and is commonly referred to as Standard Dynamical Model (Giorgini et al. 2008). To improve the integration accuracy the dynamics are scaled by Earth semi-major axis and Sun gravitational parameter (i.e., $a_{E}=1$ and $\mu_{S}=G m_{S}=1$ ). We must mention that, to obtain a full understanding of the dynamics of a body in the Solar System, other effects should be taken into account, such as: the forces due to other natural satellites and asteroids, the $J_{2}$ (and higher order harmonics of the potential) effect of the Earth and other bodies, Yarkovsky and solar radiation pressure effects (Giorgini et al. 2008; Vokrouhlický et al. 2001).

When the asteroid approaches the Earth, a different set of ODE are integrated to avoid cancellation errors associated to repetitive subtraction of Apophis and Earth's position vectors occurring across the flyby pericenter. The equation of motion, written in the J2000.0 Earth-Centered Inertial reference frame, is

$$
\ddot{\boldsymbol{r}}=G \sum_{i} \frac{m_{i}\left(\boldsymbol{r}_{i}-\boldsymbol{r}\right)}{r_{i}^{3}}+\boldsymbol{a}_{J_{2}}-\ddot{\boldsymbol{r}}_{E},
$$

where $\boldsymbol{a}_{J_{2}}$ is the effect of Earth's oblateness due to $J_{2}$ harmonic 


$$
\begin{aligned}
& a_{J_{2}, x}=\frac{J_{2} G m_{E} R_{E}^{2} x}{r^{5}}\left(1-\frac{5 z^{2}}{r^{2}}\right) \\
& a_{J_{2}, y}=\frac{J_{2} G m_{E} R_{E}^{2} y}{r^{5}}\left(1-\frac{5 z^{2}}{r^{2}}\right) \\
& a_{J_{2}, z}=\frac{J_{2} G m_{E} R_{E}^{2} z}{r^{5}}\left(3-\frac{5 z^{2}}{r^{2}}\right),
\end{aligned}
$$

and $\ddot{\boldsymbol{r}}_{E}$ is the absolute acceleration of the Earth. The same gravitational bodies of the heliocentric phase are considered, whereas relativistic corrections are neglected as their effect during a fast close encounter is negligible. In this phase the dynamics are scaled by the radius of the Earth and by the Earth gravitational parameter (i.e., $R_{E}=1$ and $\mu_{E}=G m_{E}=1$ ).

\subsection{Flow expansion}

The high order expansion of the flow of ODE can be straightforwardly obtained by evaluating any explicit numerical integration scheme within the DA framework, as explained in Sect. 3. The results presented here are obtained by applying a DA-based 8-th order RungeKutta-Fehlberg (RKF78) scheme with absolute and relative tolerance of $10^{-12}$. The integration window is June 18, 2009 to April 16, 2029, being April 13, 2029, the date of the close approach.

The nominal initial state and the associated $\sigma$ of Apophis, expressed in equinoctial variables $\boldsymbol{p}=\left(a, P_{1}, P_{2}, Q_{1}, Q_{2}, l\right)$, are taken from the Near Earth Object Dynamic Site (newton.dm.unipi.it/neodys) and summarized in Table 1. With reference to the notation of Eq. 15 , Apophis' initial condition is initialized as DA variables $\left[\boldsymbol{p}_{0}\right]=\boldsymbol{p}_{0}+3 \boldsymbol{\sigma} \delta \boldsymbol{p}_{0}$, where $3 \boldsymbol{\sigma}$ is used as a scaling factor. These variables are converted into cartesian coordinates using the relations given in Battin (1968), evaluated in the DA framework and then numerically propagated. Note that the solution of the Kepler equation, required for the computation of the eccentric longitude, is carried out by applying the DA-algorithm introduced in BernelliZazzera et al. (2009).

The nominal heliocentric trajectories of Apophis and the Earth are shown in Fig. 2 by the solid and dotted lines, respectively. Figure 3 shows a zoom of Apophis' close approach with the Earth in the geocentric reference frame. It is worth mentioning that the maximum norm of the difference between the computed trajectory and Apophis' HORIZONS ephemerides is less than $5 \times 10^{-8} \mathrm{AU}$. The mismatch is due to all different initial conditions, dynamical and ephemeris model, and integration scheme.

An analysis on the accuracy of the flow expansion is mandatory before introducing the DA-based Monte Carlo algorithm. Figure 4 shows the maximum position and velocity error of

Table 1 Apophis' equinoctial variables at 3456 MJD2000 (June $18,2009)$ and associated $\sigma$ values

\begin{tabular}{llll}
\hline & Nom value & $\sigma$ & \\
\hline$a$ & 0.922438242375914 & $2.29775 \times 10^{-8}$ & $\mathrm{AU}$ \\
$P_{1}$ & -0.093144699837425 & $3.26033 \times 10^{-8}$ & - \\
$P_{2}$ & 0.166982492089134 & $7.05132 \times 10^{-8}$ & - \\
$Q_{1}$ & -0.012032857685451 & $5.39528 \times 10^{-8}$ & - \\
$Q_{2}$ & -0.026474053361345 & $1.83533 \times 10^{-8}$ & - \\
$l$ & 88.3150906433494 & $6.39035 \times 10^{-5}$ & $\mathrm{deg}$ \\
\hline
\end{tabular}


Fig. 2 Apophis heliocentric phase trajectory

Fig. 3 Apophis close encounter trajectory
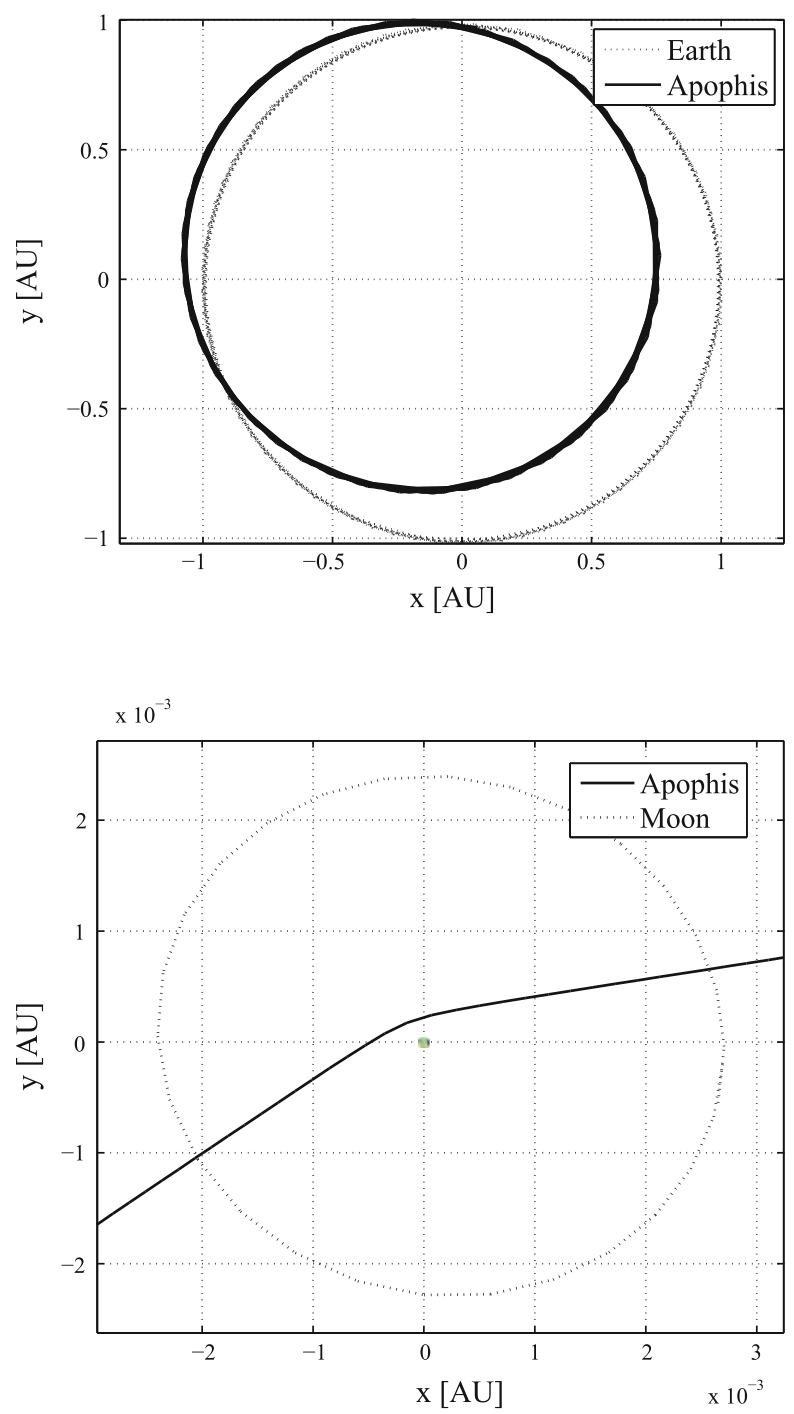

the Taylor representation of the flow at the corners of the initial set, with respect to the pointwise integration of the same points. Initial widths of 3,6 , and $9 \sigma$ and expansion orders from 1 to 5 are considered. The expansion error decreases when higher expansion orders are selected and when smaller uncertain sets are considered. The errors tend to decrease exponentially with the expansion order, until reaching a lower limit of approximately $5 \times 10^{-11}$ [AU] on position and $3 \times 10^{-10}$ [AU/day] on velocity. It is worth noticing that a fifth order expansion guarantees a gain of approximately three order of magnitude in the flow representation with respect to linear methods. This gain can be crucial when impact probability and/or resonant returns are studied. The figure clearly shows that Taylor polynomial accuracy is a function of both the expansion order and domain width. The drawback for obtaining the Taylor expansion of the flow with respect to the initial condition is the computational time to perform a single integration, as shown in Fig. 5. In this figure the ratio between the computational time of a 

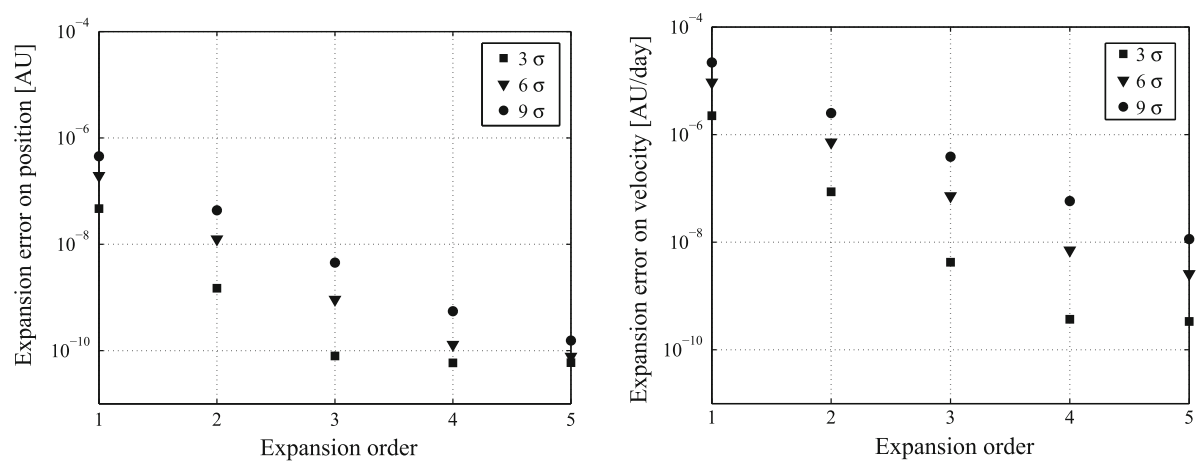

Fig. 4 Accuracy of the Taylor expansion of the flow corresponding to different expansion orders and initial uncertainty sets. Left: Position error; Right: Velocity error

Fig. 5 DA integration computational time compared to single pointwise integration

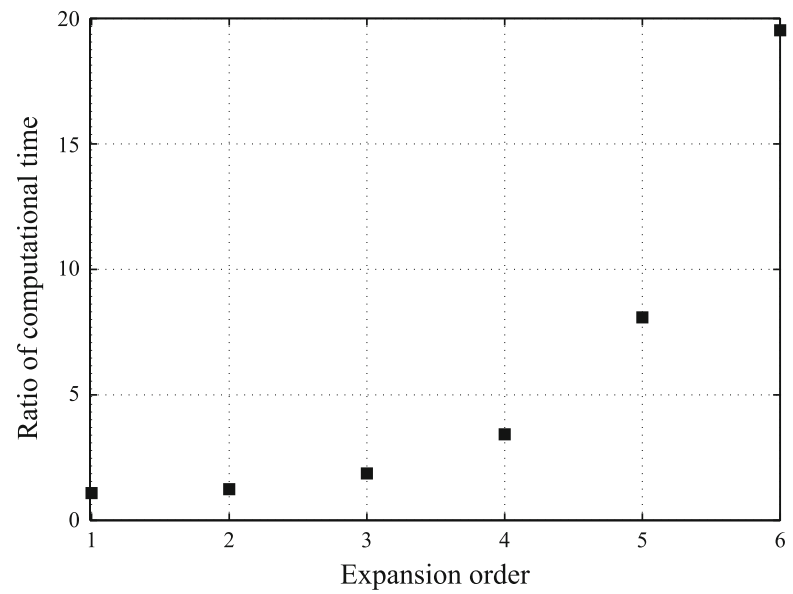

$k$-th order DA integration and a single pointwise integration is illustrated, underlining that a 5-th order integration is approximately eight times slower. On the other hand, the availability of the flow expansion enables the development of a computationally efficient Monte Carlo method, as described in the next section.

\section{DA-based Monte Carlo}

Within the dynamical models adopted and the chosen integration scheme, the asteroid reference solution has a close encounter distance from the Earth center of mass of 38161.55420 $\mathrm{km}$ at epoch 10695.907094 MJD2000. In order to evaluate the possibility of an Earth impact it is necessary to accurately propagate the statistics of the asteroid. The accurate computation of statistics in nonlinear dynamical systems often relies on Monte Carlo simulations. The algorithmic flow of a Monte Carlo simulation is:

1. Generate random samples based on the statistical distribution of the uncertainty to be propagated.

2. Run a pointwise integration of each sample in the fully nonlinear dynamics.

3. Perform the statistical analysis of the results. 
There are three critical disadvantages when using this approach:

- convergence of the statistics usually requires a large number of sample trajectories to be propagated,

- the simulation needs to be repeated for different initial distributions,

- it does not provide the user with analytical information, useful for additional analyses.

These problems affect both the computational burden associated to the Monte Carlo simulation and its validity for different statistics (Park and Scheeres 2006). The previous drawbacks become dramatic when thousands of long-term integrations are required, as for the analysis of possible NEO close encounter with the Earth (Milani et al. 2002).

In Sect. 3 it has been shown that a single DA integration delivers an arbitrary order Taylor expansion of the flow of the ODE, which is analytic. Furthermore, it has been remarked that the accuracy of the map expansion can be controlled by acting on the expansion order. For these reasons, it is possible to substitute the thousands of pointwise integrations required for classical Monte Carlo simulations with an equal number of map evaluations, i.e. fast polynomials evaluations.

The resulting DA-based Monte Carlo simulation can be summarized as:

1. Perform a single DA integration selecting the expansion order according to the demanded accuracy.

2. Generate random samples based on the statistical distribution of the uncertainty to be propagated.

3. Evaluate the flow expansion map for all the samples, requiring only fast polynomial evaluations.

4. Perform the statistical analysis of the results.

The ratio between the computational time of a DA-based Monte Carlo simulation and its pointwise counterpart is given by

$$
\frac{t_{n}+n_{s} t_{e}}{n_{s} t_{0}}
$$

where $t_{n}, t_{e}$, and $t_{0}$ are the computational times of a $k$-th order DA integration, a flow map evaluation, and a pointwise integration, respectively; and $n_{s}$ is the number of samples of the Monte Carlo simulation. The computational cost of a Taylor map evaluation depends on the expansion order, but it is negligible compared to a pointwise integration. For this reason, expression (21) can be approximated by $\frac{m}{n_{s}}$, in which $m$ is the ratio between the computational time of a $k$-th order DA integration and a pointwise integration (see Fig. 5). The value of $m$ strongly depends on the expansion order, but it is few orders of magnitude smaller than the number of samples required for a good representation of the statistics. For this reason, the ratio $\frac{m}{n_{s}}$ is small, proving that the proposed DA-based Monte Carlo simulation is computationally efficient. As an example, in Sect. 6.3, Fig. 11 will show that the computational time is reduced by a factor of at least 100 for 10000 virtual asteroids.

In case new statistics need to be propagated, it is not necessary to perform an additional DA integration as only steps $2-4$ are required. Furthermore, if the statistical analysis is performed for a different final time, the possibility of obtaining Taylor expansions with respect to the final time can be exploited (see Sect. 6.1). Moreover, as the flow expansion is analytical, an analytic framework is delivered. In conclusion, all the major drawbacks of a classical Monte Carlo approach are circumvented. These properties are better highlighted in Sect. 6 by applying the algorithm to the study of Apophis' close encounter with the Earth in 2029. 


\section{Apophis close encounter study}

As highlighted by Milani et al. (2000), impact solutions could occur for different virtual asteroids at a time when the nominal asteroid is far from the Earth. For this reason, performing DA-based Monte Carlo simulations using the Taylor expansion of the flow at the epoch of the CED of the nominal solution is not appropriate, as each virtual asteroid belonging to the set of possible initial conditions has a different close encounter epoch.

A DA-based algorithm is introduced to reduce the computation of the CED, as well as its associated epoch, to the simple evaluation of polynomials for each virtual asteroid. Being at the basis of the algorithm, a technique is illustrated in Sect. 6.1 to obtain the arbitrary order Taylor expansion of the flow of ODE also with respect to the final time. The algorithm for the computation of the Taylor expansion of the CED is then presented and applied to Apophis' case. The results obtained by running on it the DA-based Monte Carlo algorithm are illustrated.

\subsection{High order expansion of the flow in time}

The algorithm for the computation of the CED relies on the availability of the Taylor expansion of the flow of the ODE with respect to both the initial condition and the final integration time. In Sect. 3 it was shown how the flow expansion with respect to the initial condition can be computed; in the following we explain how the expansion in the final time is achieved.

Consider the ODE system

$$
\frac{\mathrm{d} \boldsymbol{x}}{\mathrm{d} t}=\boldsymbol{f}(\boldsymbol{x}, t)
$$

to be integrated from $t=t_{0}$ to $t=t_{f}$. Suppose the Taylor expansion of the flow with respect to $t_{f}$ is of interest. We first shift the starting time by introducing the variable

$$
\tilde{t}=t-t_{0} .
$$

Using the variable $\tilde{t}$, equation (22) reads

$$
\frac{\mathrm{d} \boldsymbol{x}}{\mathrm{d} \tilde{t}}=\boldsymbol{f}\left(\boldsymbol{x}, \tilde{t}+t_{0}\right),
$$

and it must be integrated from $\tilde{t}=0$ to $\tilde{t}=t_{f}-t_{0}$. Then, we introduce the variable

$$
\tau=\frac{\tilde{t}}{t_{f}-t_{0}} .
$$

Consequently,

$$
\mathrm{d} \tilde{t}=\left(t_{f}-t_{0}\right) \mathrm{d} \tau
$$

and equation (24) now reads

$$
\frac{\mathrm{d} \boldsymbol{x}}{\mathrm{d} \tau}=\left(t_{f}-t_{0}\right) \cdot \boldsymbol{f}\left(\boldsymbol{x}, t_{0}+\left(t_{f}-t_{0}\right) \tau\right),
$$

that must be integrated from $\tau=0$ to $\tau=1$. Integrating Eq. 27 from $\tau=0$ to $\tau=1$ is equivalent to integrate the original ODE (22) from $t=t_{0}$ to $t=t_{f}$. However, a major advantage can be highlighted: the final time $t_{f}$, as well as the initial time $t_{0}$, have been moved from the integration interval to the ODE right hand side, where they appear as parameters. 
Fig. 6 Apophis' distance from the Earth: comparison between the pointwise integration and the Taylor expansion of the flow with respect to the final integration epoch

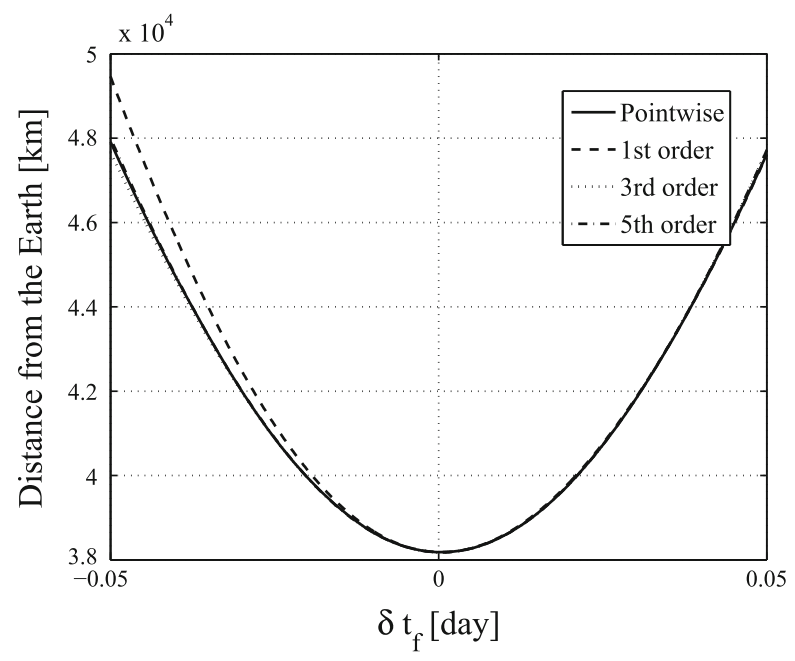

This allows the flow of the ODE to be expanded also with respect to the final epoch. More specifically, the final time $t_{f}$ can be initialized as a DA variable:

$$
\left[t_{f}\right]=t_{f}+\delta t_{f}
$$

in which $t_{f}$ is the reference final epoch. Then, Eq. 27 is integrated from $\tau=0$ to $\tau=1$. Carrying out all the algebraic operations involved in the integration scheme in the DA framework allows the dependence of the solution on $\delta t_{f}$ to be carried forward all throughout the integration. The result at time $\tau=1$ is

$$
\left[\boldsymbol{x}_{\boldsymbol{f}}\right]=\boldsymbol{x}_{f}+\mathcal{M}_{\boldsymbol{x}_{f}}\left(\delta t_{f}\right)
$$

i.e., the Taylor expansion of the final solution with respect to the final time. Note that the Taylor expansion of the solution with respect to the initial condition and the final time can be obtained by initializing both $\boldsymbol{x}_{0}$ and $t_{f}$ as DA variables.

The previous technique can be immediately applied to the integration of Apophis' motion in order to obtain the Taylor expansion of the final position and velocity with respect to the final epoch. In order to show the performances of the algorithm, set the initial condition $\boldsymbol{x}_{0}$ to be the nominal Apophis'position and velocity at the initial epoch $t_{0}$, and choose the reference epoch $t_{f}$ to be the epoch of the close encounter for the nominal Apophis' initial condition. The DA-based RKF78 scheme is then used to expand the solution of the ODE governing the asteroid motion with respect to $t_{f}$. Figure 6 compares the nominal Apophis' distance from the Earth obtained through a pointwise integration with that computed by evaluating the Taylor expansion of Apophis' position with respect to $t_{f}$, in the interval $t_{f}-0.05$ days $<t_{f}<t_{f}+0.05$ days. More specifically, the results corresponding to three different expansion orders are illustrated. As it can be seen, the accuracy of the Taylor representation increases with the expansion order.

The accuracy of the Taylor representations is better assessed in Fig. 7. In particular, the absolute difference between the results of the pointwise integration and Taylor expansion evaluation is plotted. As expected, the error is maximum at the boundary of the interval, whereas it decreases toward the center; i.e., toward the reference epoch of the Taylor expansions. The fifth order expansion in time guarantees an accuracy comparable with that in the transversal coordinates for a time interval consistent with the analysis proposed in Sect. 6.3. 
Fig. 7 Analysis of the error of the Taylor expansion of the flow with respect to the final integration epoch for Apophis' position

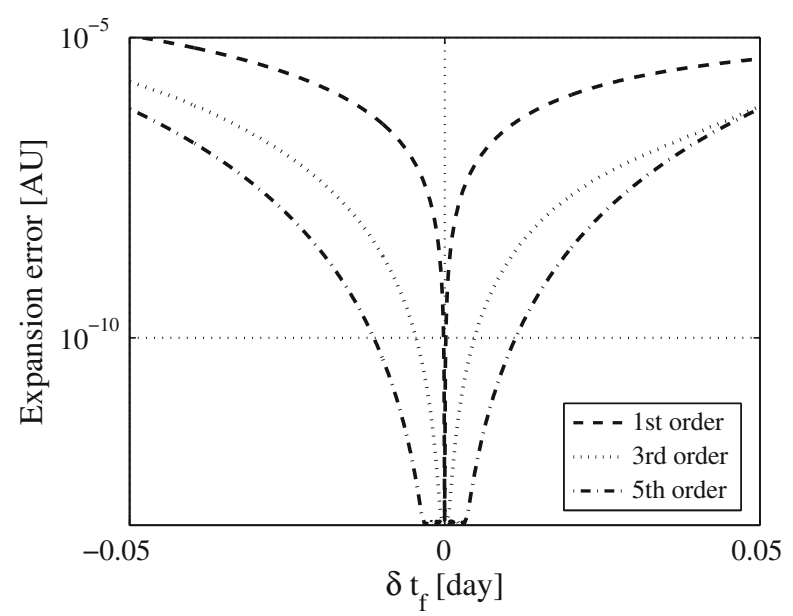

The accuracy analyses presented in this section and in Sect. 4.2 are based on checking how well the coordinates are predicted by the flow map versus direct integration. It is worth mentioning that in principle it is possible to get rigorous error bounds on the maps by using Taylor models (TM) and Taylor model integrators (Hoefkens et al. 2003). As the outcome of a TM integration is the validated enclosure of the flow of the dynamics, a single integration (without any further additional analysis) would suffice for the study of Apophis' close encounter. On the other hand, the use of TM requires a careful coding of both the dynamical model and the ephemeris function in order to efficiently compute the rigorous enclosure of the flow during the asteroid's close encounter phase. This aspect is currently under investigation by the authors.

\subsection{CED algorithm}

Let us suppose the close approach of the nominal asteroid occurs at the epoch $t_{f}$, and consider the integration of the asteroid dynamics in the form (27) from $t=t_{0}$ to $t=t_{f}$. Initialize the initial state and the final integration epoch as DA variables; i.e.,

$$
\begin{aligned}
& {\left[x_{0}\right]=x_{0}+\delta x_{0}} \\
& {\left[t_{f}\right]=t_{f}+\delta t_{f},}
\end{aligned}
$$

where $\boldsymbol{x}_{0}$ is the initial condition corresponding to the nominal asteroid. Using the DA-based RKF78 integrator and the technique introduced in Sect. 6.1 obtain the map

$$
\left[\boldsymbol{x}_{f}\right]=\boldsymbol{x}_{f}+\mathcal{M}_{\boldsymbol{x}_{f}}\left(\delta \boldsymbol{x}_{0}, \delta t_{f}\right) .
$$

The map (31) is the $k$-th order Taylor expansion of the flow of (27) with respect to the initial condition and the final epoch about their nominal values $\boldsymbol{x}_{0}$ and $t_{f}$. Based on a mere DAbased computation, the final solution $\boldsymbol{x}_{f}$ can be used to compute the Taylor expansion of distance from the Earth

$$
\left[d_{f}\right]=d_{f}+\mathcal{M}_{d_{f}}\left(\delta \boldsymbol{x}_{0}, \delta t_{f}\right) .
$$

More specifically, map (32) describes how the distance varies depending on the virtual asteroid and the final integration epoch. 
Using the derivation operator available in the DA framework, the Taylor expansion of the derivative $d_{f}^{\prime}=\mathrm{d}\left(d_{f}\right) / \mathrm{d} t_{f}$ can be obtained

$$
\left[d_{f}^{\prime}\right]=d_{f}^{\prime}+\mathcal{M}_{d_{f}^{\prime}}\left(\delta \boldsymbol{x}_{0}, \delta t_{f}\right) .
$$

The constant part of the map (33), $d_{f}^{\prime}$, is the derivative of the distance from the Earth of the nominal solution at its close encounter; i.e., at CED epoch. Consequently, this is a stationary point for the nominal solution, and $d_{f}^{\prime}=0$. Then, the map (33) reduces to

$$
\delta d_{f}^{\prime}=\mathcal{M}_{d_{f}^{\prime}}\left(\delta \boldsymbol{x}_{0}, \delta t_{f}\right),
$$

in which we omit the bracket operator for the sake of a simpler notation. Consider the map

$$
\left(\begin{array}{l}
\delta d_{f}^{\prime} \\
\delta \boldsymbol{x}_{0}
\end{array}\right)=\left(\begin{array}{c}
\mathcal{M}_{d_{f}^{\prime}} \\
\mathcal{I}_{\boldsymbol{x}_{0}}
\end{array}\right)\left(\begin{array}{l}
\delta \boldsymbol{x}_{0} \\
\delta t_{f}
\end{array}\right),
$$

which is built by concatenating $\mathcal{M}_{d_{f}^{\prime}}$ with the identity map for $\delta x_{0}$. Map (35) can now be inverted to obtain

$$
\left(\begin{array}{l}
\delta \boldsymbol{x}_{0} \\
\delta t_{f}
\end{array}\right)=\left(\begin{array}{c}
\mathcal{M}_{d_{f}^{\prime}} \\
\mathcal{I}_{\boldsymbol{x}_{0}}
\end{array}\right)^{-1}\left(\begin{array}{c}
\delta d_{f}^{\prime} \\
\delta \boldsymbol{x}_{0}
\end{array}\right) .
$$

This is a full nonlinear map inversion that is obtained by applying the algorithm illustrated in Berz (1999b). This algorithm reduces the map inversion problem to the solution of an equivalent fixed point problem, which can be solved with a fixed amount of effort in the DA setting.

Map (32) is then concatenated to the identity map for $\delta t_{f}$ to obtain

$$
\left(\begin{array}{l}
d_{f} \\
\delta t_{f}
\end{array}\right)=\left(\begin{array}{c}
\mathcal{M}_{d_{f}} \\
\mathcal{I}_{t_{f}}
\end{array}\right)\left(\begin{array}{l}
\delta \boldsymbol{x}_{0} \\
\delta t_{f}
\end{array}\right) .
$$

Map (37) can now be composed with map (36) to obtain

$$
\left(\begin{array}{c}
d_{f} \\
\delta t_{f}
\end{array}\right)=\left(\begin{array}{c}
\mathcal{M}_{d_{f}} \\
\mathcal{I}_{t_{f}}
\end{array}\right) \circ\left(\begin{array}{c}
\mathcal{M}_{d_{f}^{\prime}} \\
\mathcal{I}_{\boldsymbol{x}_{0}}
\end{array}\right)^{-1}\left(\begin{array}{c}
\delta d_{f}^{\prime} \\
\delta \boldsymbol{x}_{0}
\end{array}\right),
$$

which relates $d_{f}$ and $\delta t_{f}$ to the displacements of the derivative of the final distance $\delta d_{f}^{\prime}$ and of the initial state vector of the virtual asteroid $\delta \boldsymbol{x}_{0}$ from their values. As for the reference value $d_{f}^{\prime}=0$, the necessary condition for CED computation is

$$
\delta d_{f}^{\prime}=0 .
$$

Substituting into (38) yields

$$
\left(\begin{array}{c}
d_{f}^{*} \\
\delta t_{f}^{*}
\end{array}\right)=\left(\begin{array}{c}
\mathcal{M}_{d_{f}} \\
\mathcal{I}_{t_{f}}
\end{array}\right) \circ\left(\begin{array}{c}
\mathcal{M}_{d_{f}^{\prime}} \\
\mathcal{I}_{\boldsymbol{x}_{0}}
\end{array}\right)^{-1}\left(\begin{array}{c}
0 \\
\delta \boldsymbol{x}_{0}
\end{array}\right) .
$$

Eventually, map (40) delivers the desired explicit relation between the CED $\left(d_{f}{ }^{*}\right)$ and the epoch at which it is reached $\left(t_{f}+\delta t_{f}{ }^{*}\right)$ with the displacement $\delta \boldsymbol{x}_{0}$ in terms of Taylor polynomials. Given any virtual asteroid belonging to the initial set (which corresponds to a specific value of the displacement $\delta \boldsymbol{x}_{0}$ ), the simple evaluation of the polynomials in (40) delivers the $\mathrm{CED}$ and the epoch at which it is reached. In this way, the problem highlighted by Milani et al. (2000) is solved. 


\subsection{CED statistical analysis}

The DA-based Monte Carlo simulation introduced in Sec. 5 is run on map (40) to perform the nonlinear mapping of the initial uncertainties on the close encounter distance (CED). More specifically, 10000 virtual asteroids are generated with a normal random distribution with mean value and standard deviation as in Table 1. For each sample, the displacement with respect to the nominal initial conditions is computed and map (40) is evaluated to obtain its CED and the associated epoch. The result is reported in Fig. 8(left) in terms of probability distribution for the CED. The analysis of the results shows that the mean value is 38161.54 $\mathrm{km}$ with a standard deviation of $492.1 \mathrm{~km}$, thus the possibility of having an Earth impact in 2029 is ruled out.

For the same virtual asteroids, map (40) is also evaluated to obtain the close encounter epochs. The result is presented in Fig. 8(right) in terms of the probability distribution of the displacement $\delta t_{f}{ }^{*}$ from the nominal epoch $t_{f}$. The maximum displacement is of the order of $30 \mathrm{~s}$, which is compatible with the accuracy of the Taylor expansion with respect to the final epoch shown in Fig. 7. In Fig. 9 the 10000 virtual asteroids are plotted in the CED- $\delta t_{f}^{*}$ plane.
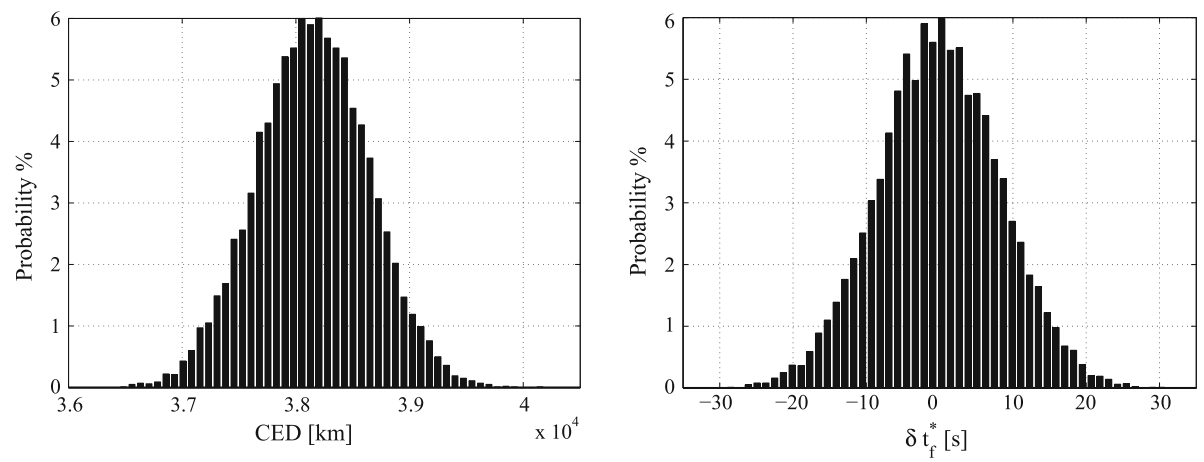

Fig. 8 Monte Carlo analysis of virtual asteroids close encounter distances (CED). Left: The distances; Right: The epochs

Fig. 9 CED vs $\delta t_{f}^{*}$ for 10000 virtual asteroids

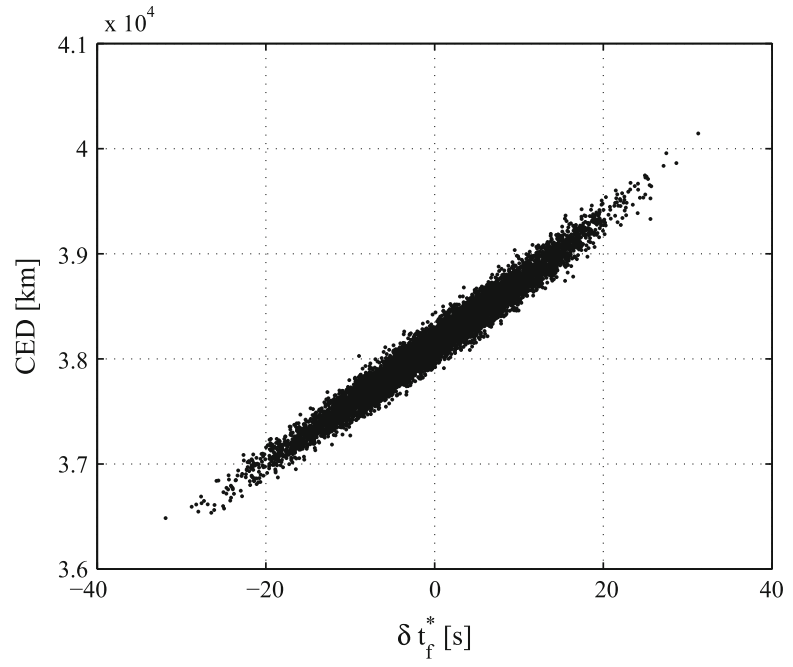


Fig. 10 Accuracy of the CED algorithm: virtual asteroids actual trajectories
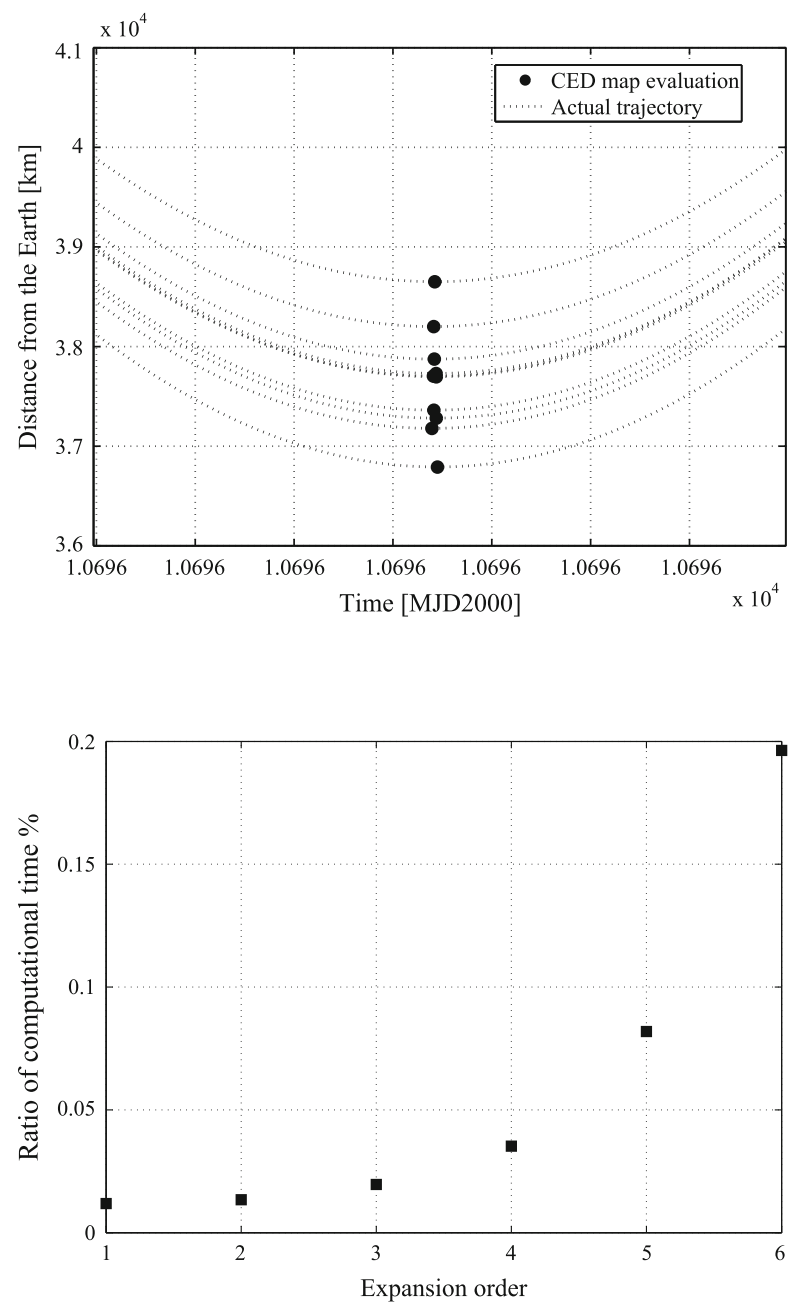

Fig. 11 Percentage of computational time required by a DA-based Monte Carlo run versus a classical Monte Carlo simulation for 10000 virtual asteroids

For the sake of completeness, an accuracy analysis of the results is presented in Fig. 10. Ten virtual asteroids are randomly selected from the initial set. For each virtual asteroid, the minimum distance and the corresponding epoch, resulting from map (40), are reported in the figure. Then, a pointwise integration of the motion of each asteroid is performed to obtain the profile of Earth's distances shown in the dotted lines. Although the accuracy on the identification of the epoch of the close encounter is not clearly visualized, due to the very little displacement in $t_{f}{ }^{*}$, it is clearly shown that the algorithm is able to accurately identify the CED values of the resulting trajectories. Figure 11 concludes the analysis by showing the ratio of the computational time between the proposed DA-based Monte Carlo simulation and its pointwise counterpart as a function of the expansion order when 10000 virtual asteroids are considered. It is apparent that the drawback of the higher computational cost required by a DA integration is rewarded by the significant time saving achieved by substituting 10000 pointwise integrations with the same number of polynomials evaluations. 


\section{Conclusions}

The paper introduced a Monte Carlo simulation based on the high order Taylor expansion of the flow of ODE, enabled by the use of differential algebra. Being based on the replacement of pointwise integrations with fast evaluation of polynomials, the proposed algorithm guarantees significant computational time savings. The accuracy of the algorithm can be suitably tuned by varying the flow expansion order. Furthermore, the availability of analytic Taylor expansions and the use of DA embedded tools as map inversion, composition, and derivation allow the user to compute arbitrary order maps of the quantities on which the statistical analysis is performed; thus, the algorithm is not limited to the flow of ODE. More specifically, a technique for the automatic computation of both CED and CED epochs for all the virtual asteroids belonging to the initial uncertainty cloud has been developed. The efficiency and effectiveness of the methods are proven by applying them to the analysis of Apophis' close encounter with the Earth occurring in April 2029. In particular, it is shown that

- the nonlinear mapping of uncertainties can be performed for any complex and arbitrary dynamics, even when long-term integrations are required;

- a fifth order expansion increases the accuracy of the computation of the CED by approximately two orders of magnitude with respect to classical linear methods;

- the expansion in time allows for the proper identification of the CED epoch for all the virtual asteroids.

As an additional result, the occurrence of an impact with the Earth in April 2029 can be ruled out.

Acknowledgments This work was inspired by Park and Scheeres (2006) and was partially conducted under the ESA Ariadna study "NEO Encounter 2029". The authors are grateful to Dario Izzo for having encouraged the work and to Jon Giorgini for his precious comments.

\section{References}

Battin, R.H.: An Introduction to the Mathematics and Methods of Astrodynamics. AIAA Education Series, New York (1968)

Berges, J., Rousseau, S., Perot E.: A Numerical Predictor-Corrector Guidance Algorithm for the Mars Sample Return Aerocapture. AAAF, 14-66, March (2001)

Bernelli-Zazzera, F., Berz, M., Lavagna, M., Makino, K., Armellin, R., Di Lizia, P., Jagasia, R., Topputo, F.: NEO Encounter 2029: Orbital Prediction via Differential Algebra and Taylor Models. Final Report, Ariadna id: 08-4303, Contract No. 20271/06/NL/HI (2009)

Berz, M.: The new method of TPSA algebra for the description of beam dynamics to high orders. Technical Report AT-6:ATN-86-16, Los Alamos National Laboratory (1986)

Berz, M.: The method of power series tracking for the mathematical description of beam dynamics. Nuclear Instrum. Meth. A258, 431 (1987)

Berz, M.: High-order computation and normal form analysis of repetitive systems. Phys. Particle Accel. AIP 249, 456 (1991)

Berz, M., Joh, K., Nolen, J.A., Sherrill, B.M., Zeller, A.F.: Reconstructive correction of aberration in nuclear particle spectrographs. Phys. Rev. C 47, 537-544 (1993)

Berz, M.: Differential Algebraic Techniques. Entry in Handbook of Accelerator Physics and Engineering. World Scientific, New York (1999a)

Berz, M.: Modern Map Methods in Particle Beam Physics. Academic Press, London (1999b)

Berz M., Makino K.: COSY INFINITY version 9 reference manual. MSU Report MSUHEP-060803, Michigan State University, East Lansing, MI 48824, pp. 1-84 (2006)

Chesley, S.R., Milani, A.: An automatic earth-asteroid collision monitoring system. Bull. Am. Astron. Soc. 32, 682 (2000) 
Chodas, P.W., Yeomans, D.K.: Predicting close approaches and estimating impact probabilities for near-Earth projects. AAS/AIAA Astrodynamics Specialists Conference, Girdwood, Alaska (1999)

Crassidis, J.L., Junkins, J.L.: Optimal Estimation of Dynamics Systems. pp. 243-410. CRC Press LLC, Boca Raton, FL (2004)

Di Lizia, P., Armellin, R., Lavagna, M.: Application of high order expansions of two-point boundary value problems to astrodynamics. Celest. Mech. Dyn. Astron. 102, 355-375 (2008)

Erdelyi, B., Bandura, L., Nolen, J., Manikonda, S.: Code development for next-generation high-intensity large acceptance fragment separators. In: Proceedings of PAC07, Albuquerque, New Mexico, USA (2007)

Giorgini, J.D., Benner, L.A.M., Ostro, S.J., Nolan, M.C., Busch, M.W.: Predicting the earth encounters of (99942) apophis. Icarus 193, 1-19 (2008)

Griffith, T.D., Turner, J.D., Vadali, S.R., Junkins, J.L.: Higher order sensitivities for solving nonlinear two-point boundary-value problems. In: AIAA/AAS Astrodynamics Specialist Conference and Exhibit, Providence, Rhode Island, August 16-19 (2004)

Hoefkens, J., Berz, M., Makino, K.: Controlling the wrapping effect in the solution of ODEs for asteroids. Reliable Comput. 8, 21-41 (2003)

Junkins, J., Akella, M., Alfriend, K.: Non-Gaussian error propagation in orbit mechanics. J. Astronaut. Sci. 44, 541-563 (1996)

Junkins, J., Singla, P.: How nonlinear is it? A tutorial on nonlinearity of orbit and attitude dynamics. J. Astronaut. Sci. 52, 7-60 (2004)

Maybeck, P.S.: Stochastic Models, Estimation, and Control. pp. 159-271. Academic Press, New York (1982)

Milani, A., Chesley, S.R., Valsecchi, G.B.: Asteroid close encounters with Earth: Risk assessment. Planet. Space Sci. 48, 945-954 (2000)

Milani, A., Chesley, S.R., Chodas, P.W., Valsecchi, G.B.: Asteroid close approaches: analysis and potential impact detection. Asteroids III, pp. 89-101 (2002)

Montenbruck, O., Gill, E.: Satellite Orbits. pp. 257-291. 2nd edn. Springer, New York (2001)

Park, R., Scheeres, D.: Nonlinear mapping of Gaussian statistics: theory and applications to spacecraft trajectory design. J. Guidance Control Dyn. 29, 1367-1375 (2006)

Park, R.S., Scheeres, D.J.: Nonlinear semi-analytic methods for trajectory estimation. J. Guidance Control Dyn. 30, 1668-1676 (2007)

Seidelmann, P.K.: Explanatory Supplement to the Astronomical Almanac. University Science Books, Mill Valley, California (1992)

Vokrouhlický, D., Chesley, S.R., Milani, A.: On the observability of radiation forces acting on near-earth a steroids. Celest. Mech. Dyn. Astron. 81, 149-165 (2001) 